\title{
Resection versus biopsy of glioblastomas in eloquent brain areas
}

\author{
Lumir Hrabaleka, Ondrej Kalita ${ }^{a}$, Miroslav Vaverka ${ }^{a}$, Marian Hajduch ${ }^{b}$, Miloslava Zlevorovac, Jiri Ehrmann Jrd, \\ Eva Cechakova ${ }^{g}$, Milan Adamus ${ }^{e}$, Vlastimil Novaka, Katerina Langova ${ }^{f}$
}

\begin{abstract}
Aim. The aim of this study was to compare resection and biopsy of glioblastoma (GBM) in eloquent brain areas (EBA). Methods. This was a prospective evaluation of 38 patients with GBM in EBA. 22 were treated by surgical resection and 16 by biopsy. Preoperative KPS, neurological status and size of lesion on MRI were assessed. One week and three months postoperatively KPS, neurological status and Performance Status (PS) WHO were evaluated. Extent of resection (EOR) and overall survival (OS) were described. Overall mean age of the patients was 64.3 years, the mean lesion size in the resection group was $47.7 \mathrm{~mm}$ and in the biopsy group $51.0 \mathrm{~mm}$.

Results. Worsening or development of permanent neurological deficits 3 months after surgery were significantly lower in the resection group (23\%), than the biopsy group (94\%). In the resection group the median pre and postoperative KPS three months after surgery was 80.0. In the biopsy group the median pre and postoperative KPS was 68.1 one week after the procedure. In the resection group, 3 months after surgery, the median PS was 1, in the biopsy group one week after surgery the median PS was 2. The difference was statistically insignificant. The mean OS after resection was 12.2 months, and after biopsy 3.5 months. The difference was highly statistically significant. The mean EOR was $90 \%$. Conclusion. This is the first prospective study, to our knowledge, that compares the results of resection and biopsy of primary GBM in EBA. For patients in good clinical condition with tumors in or near EBA, recommended is as radical resection of GBM as possible.
\end{abstract}

Key words: glioblastoma multiforme, eloquent brain area, resection, biopsy

Received: March 17, 2013; Accepted with revision: July 1, 2013; Available online: July 29, 2013 http://dx.doi.org/10.5507/bp.2013.052

\begin{abstract}
${ }^{a}$ Department of Neurosurgery, Faculty of Medicine and Dentistry, Palacky University Olomouc and University Hospital Olomouc, Czech Republic

'Laboratory of Experimental Medicine, Faculty of Medicine and Dentistry, Palacky University Olomouc and University Hospital Olomouc 'Department of Oncology, Faculty of Medicine and Dentistry, Palacky University Olomouc and University Hospital Olomouc ${ }^{d}$ Department of Pathology, Faculty of Medicine and Dentistry, Palacky University Olomouc and University Hospital Olomouc ${ }^{e}$ Department of Anesthesiology and Intensive Care Medicine, Faculty of Medicine and Dentistry, Palacky University Olomouc and University Hospital Olomouc

${ }^{f}$ Department of Medical Biophysics, Faculty of Medicine and Dentistry, Palacky University Olomouc and University Hospital Olomouc ${ }^{9}$ Department of Radiology, Faculty of Medicine and Dentistry, Palacky University Olomouc and University Hospital Olomouc Corresponding author: Lumir Hrabalek, e-mail: lumir.hrabalek@seznam.cz
\end{abstract}

\section{INTRODUCTION}

Patients with glioblastoma multiforme (GBM) have two initial surgical management options; biopsy or resection. Some situations demand surgical resection and in others, biopsy is the only choice. The goal of GBM surgery is to obtain tissue samples for histological diagnosis or achieve maximum extent of surgical resection (cytoreduction, reduction in mass) without causing new neurological deficits. Eloquent brain areas (EBA), resections in particular, are frequently terminated before total tumor removal to avoid this outcome. A retrospective analysis of Lacroix et al. combining 416 patients with newly diagnosed or recurrent GBM, concluded that more than $98 \%$ extent of resection (EOR) is necessary to prolong survival significantly ${ }^{1}$. In the modern era, this report serves as a critical reference for the neurosurgical community, justifying the "all-or-none" approach commonly practiced in the surgical management of GBM. This was the reason for indicating needle biopsy in cases that precluded radical tumor resection, especially in critical brain areas. It remains today, that there is no consensus on the treatment for tumors located in or near EBA, whether incomplete resection or only biopsy is indicated.

We carried out the following study to compare the outcome of resection and biopsy of GBM in EBA.

\section{MATERIAL AND METHODS}

All patients who had undergone surgery (surgical resection or needle biopsy) for intracranial primary GBM in or near EBA from January 2007 to January 2009 were identified and prospectively assessed. Eloquent brain areas included dominant superior temporal, supramarginal and angular gyri (language cortex), dominant and nondominant precentral and postcentral gyri (primary motor/ sensory cortices), supplementary motor area and/or basal ganglia/internal capsule. The designation of the presumed motor and language eloquent areas was based on preoperative diagnostic anatomical Magnetic Resonance Imaging (MRI) in all cases. In cases of tumors not clearly localized 
in EBA, a functional MRI (f-MRI) was performed later (after 1-3 weeks). Patients with prior resections, tumors crossing the corpus callosum, and infratentorial gliomas were excluded. Those who were considered unfit to undergo surgical resection were also excluded.

The Karnofsky Performance Scale (KPS) index was used to classify the preoperative functional status. The preoperative neurological status was also evaluated. Size of lesion (the largest diameter based on T1-contrastweighted images on MRI) was recorded.

The decision to use surgical resection (resection group) versus needle biopsy (biopsy group) was determined subjectively by experienced neurosurgeons together with an oncologist (usually according patient status). Surgical resection of GBM was performed under general anesthesia as open craniotomy using operating microscopy, but without other supporting methods (navigation, intraoperative neuromonitoring etc.) in 22 patients. Needle biopsy was performed using CT-guided stereotaxy (Leksell Stereotactic System, Electa, Sweden) under local anesthesia in 16 patients. The pathology was determined by a neuropathologist in all cases and was based on the 2007 World Health Organization (WHO) criteria. The degree of resection was verified from the MRIs obtained 48 hours after surgical resection. Resection was considered as gross total resection (GTR) if no residual enhancement was noted on postoperative MRI (EOR more than 99\%), near total resection (NTR) if only rim enhancement of resection cavity was found on postoperative MRI (EOR 95-99\%), subtotal resection (STR) if residual nodular enhancement was detected on postoperative MRI (EOR 90-95\%) and partial resection (PR) with EOR less than $90 \%$. The patients in the biopsy group were controlled by CT only.

Evaluation of postoperative neurological status (motor deficit, language deficit or seizures) was done one week and three months after the procedure in the resection group and one week, and in the interval from one to three months in the biopsy group. KPS and PS (Performance Status) WHO were recorded one week (biopsy group) and three months (resection group) after surgery. PS WHO is described in Table 1. Changes in KPS (difference between KPS before and after the procedure) in each group were statistically evaluated. PS WHO after surgery in both groups were statistically compared.

MRI and clinical follow-up were done every 3-6 months. For all patients in good clinical condition, the oncologist indicated concomitant chemo-radiotherapy (60 Gy at 2 Gy per dose and five doses a week, and temozolomid) followed by adjuvant chemotherapy (temozolomid). Radiotherapy was initiated within 3-4 weeks of surgery or biopsy.

We recorded the date of death and evaluated overall survival (OS) time (all evaluated patients expired).

\section{Statistical analysis}

The Shapiro-Wilk test of normality was used to test whether the distribution of variables followed a gaussian pattern. Normally distributed data were described as means and standard deviation (SD) and compared with
Table 1. Performance Status.

\begin{tabular}{lc}
\hline Patients findings & Status \\
\hline No symptoms & 0 \\
Patient symptomatic but ambulatory & 1 \\
Patient bedridden less than half the day & 2 \\
Patient bedridden half the day or longer & 3 \\
Patient chronically bedridden and requires & 4 \\
assistance with activities of daily living & \\
\hline
\end{tabular}

a $t$-test. Non-normally distributed data were described using medians and interquartile range (IQR) and compared with the Mann-Whitney $U$-test and Wilcoxon signed-rank test. Categorical data were analyzed with the Fisher's exact test. Statistical difference of survival between patients who underwent surgical resection and needle biopsy was compared by the log-rank test.

All tests were two-sided, and a $P$ value of $<0.05$ was considered significant. The Confidence intervals (CIs) were calculated at the 95\% level. All statistical analyses were conducted with SPSS (version. 15.0.1; SPSS Inc., Chicago, IL, USA) software.

\section{Study group}

Altogether 38 patients were included in the study. There were 15 female and 23 male patients (resection group 8 female and 14 male patients, biopsy group 7 female and 9 male patients). There was no significant difference in gender ratio in the groups (Fisher's exact test, $P=0.743$ ).

The overall mean age of patients was 64.3 years (SD 10.2) ranging from 43-89 years. In the resection group, the mean age was 62.5 years (SD 7.1), range 49-77 years. Patients treated by biopsy had a mean age of 66.9 years (SD 13.1), range 43-89 years. There was no significant difference in age between the two groups (Student $t$-test, $P=0.239$ ).

The mean lesion size in the resection group was 47.7 $\mathrm{mm}$ (SD 13.0) and in the biopsy group $51.0 \mathrm{~mm}$ (SD 11.9). The difference was not statistically significant (Student $t$-test, $P=0.433$ ).

The median preoperative KPS was 80 (IQR 70-82.5) in the resection group and 60 (IQR 52.5-87.5) in the biopsy treated group. We compared KPS using Mann-Whitney $U$-test and there was no statistically significant difference between the groups $(P=0.057)$.

The groups were homogenous in terms of brain location (EBA), gender, age, size of lesion and clinical status (preoperative KPS) and were thus comparable (despite of almost significant preoperative KPS difference).

\section{RESULTS}

Table 2 shows the details of patients: age, gender, preoperative KPS, postoperative KPS, PS WHO, OS, size of lesion, radiotherapy, chemotherapy in resection group 
Table 2. Details of patients.

\begin{tabular}{|c|c|c|c|c|c|c|c|c|c|c|}
\hline $\begin{array}{l}\text { Resection } \\
\text { group }\end{array}$ & Age & Gender & $\begin{array}{l}\text { KPS } \\
\text { preop }\end{array}$ & $\begin{array}{c}\text { KPS } \\
\text { postop }\end{array}$ & PS WHO & OS & Size & Radioth & Chemoth & EOR \\
\hline 1 & 68 & $\mathrm{M}$ & 60 & 80 & 1 & 27 & 52 & yes & yes & PR \\
\hline 2 & 73 & M & 80 & 70 & 1 & 1 & 67 & no & no & STR \\
\hline 3 & 61 & M & 80 & 80 & 2 & 25 & 61 & yes & yes & STR \\
\hline 4 & 62 & $\mathrm{~F}$ & 90 & 90 & 1 & 18 & 23 & yes & yes & PR \\
\hline 5 & 67 & $\mathrm{~F}$ & 80 & 90 & 1 & 49 & 51 & yes & yes & NTR \\
\hline 6 & 58 & $\mathrm{~F}$ & 90 & 90 & 1 & 8 & 49 & yes & yes & NTR \\
\hline 7 & 49 & M & 70 & 60 & 2 & 7 & 64 & yes & yes & STR \\
\hline 8 & 62 & M & 80 & 50 & 3 & 4 & 52 & yes & no & STR \\
\hline 9 & 67 & $\mathrm{M}$ & 70 & 70 & 2 & 6 & 55 & yes & yes & PR \\
\hline 10 & 55 & $\mathrm{M}$ & 70 & 80 & 2 & 10 & 58 & yes & yes & PR \\
\hline 11 & 61 & $\mathrm{~F}$ & 60 & 50 & 3 & 8 & 25 & yes & yes & GTR \\
\hline 12 & 65 & $\mathrm{M}$ & 80 & 80 & 1 & 10 & 48 & yes & yes & NTR \\
\hline 13 & 71 & $\mathrm{~F}$ & 80 & 80 & 2 & 3 & 51 & yes & no & STR \\
\hline 14 & 65 & $\mathrm{M}$ & 90 & 90 & 0 & 11 & 38 & yes & yes & NTR \\
\hline 15 & 57 & $\mathrm{M}$ & 80 & 70 & 2 & 16 & 42 & yes & yes & NTR \\
\hline 16 & 77 & $\mathrm{M}$ & 60 & 60 & 2 & 5 & 19 & yes & yes & STR \\
\hline 17 & 60 & $\mathrm{M}$ & 80 & 80 & 1 & 9 & 54 & yes & yes & NTR \\
\hline 18 & 67 & $\mathrm{M}$ & 80 & 80 & 1 & 10 & 65 & yes & yes & STR \\
\hline 19 & 56 & $\mathrm{~F}$ & 80 & 90 & 1 & 15 & 46 & yes & yes & GTR \\
\hline 20 & 49 & $\mathrm{~F}$ & 100 & 90 & 1 & 10.5 & 42 & yes & yes & PR \\
\hline 21 & 67 & $\mathrm{M}$ & 90 & 30 & 3 & 4 & 49 & no & no & PR \\
\hline 22 & 58 & $\mathrm{~F}$ & 80 & 90 & 1 & 11 & 39 & yes & yes & GTR \\
\hline \multicolumn{11}{|l|}{$\begin{array}{l}\text { Biopsy } \\
\text { group }\end{array}$} \\
\hline 1 & 57 & $\mathrm{~F}$ & 90 & 90 & 1 & 2 & 50 & yes & no & \\
\hline 2 & 67 & $\mathrm{M}$ & 60 & 60 & 2 & 2 & 75 & yes & no & \\
\hline 3 & 86 & $\mathrm{~F}$ & 50 & 50 & 3 & 1 & 71 & no & no & \\
\hline 4 & 60 & $\mathrm{M}$ & 50 & 50 & 3 & 4 & 65 & yes & no & \\
\hline 5 & 78 & $\mathrm{~F}$ & 60 & 60 & 2 & 5 & 40 & yes & no & \\
\hline 6 & 57 & $\mathrm{~F}$ & 70 & 70 & 2 & 2 & 46 & yes & yes & \\
\hline 7 & 55 & $\mathrm{M}$ & 60 & 60 & 2 & 4 & 64 & yes & no & \\
\hline 8 & 84 & $\mathrm{M}$ & 90 & 90 & 1 & 2 & 47 & yes & no & \\
\hline 9 & 89 & $\mathrm{M}$ & 80 & 80 & 2 & 5 & 37 & no & no & \\
\hline 10 & 58 & $\mathrm{M}$ & 90 & 90 & 1 & 4 & 48 & yes & yes & \\
\hline 11 & 68 & $\mathrm{~F}$ & 50 & 50 & 3 & 1 & 39 & no & no & \\
\hline 12 & 68 & $\mathrm{~F}$ & 40 & 40 & 2 & 1.5 & 46 & no & no & \\
\hline 13 & 59 & $\mathrm{~F}$ & 60 & 60 & 2 & 4 & 37 & no & no & \\
\hline 14 & 61 & $\mathrm{M}$ & 80 & 80 & 2 & 2 & 55 & yes & no & \\
\hline 15 & 80 & $\mathrm{M}$ & 60 & 60 & 2 & 1 & 49 & no & no & \\
\hline 16 & 43 & $\mathrm{M}$ & 100 & 100 & 0 & 15.1 & 47 & yes & yes & \\
\hline
\end{tabular}

Age: age at time of surgery or biopsy (years),

Gen: gender (F-female/M-male),

KPS preop: Karnofsky Performance Scale before procedure (0-100\%),

KPS postop: Karnofsky Performance Scale three months (surgery group) or one week (biopsy group) after procedure (0-100\%),

PS: Performance Status WHO (0-4),

OS: overall survival time (months),

Size: maximal size of tumor $(\mathrm{mm})$,

RTR: radiotherapy (yes/no),

ChTR: chemotherapy (yes/no),

EOR: extent of resection (GTR-gross total resection, NTR-near total resection, STR-subtotal resection, PR-partial resection). 
(22 patients) and biopsy group (16 patients). EOR is only described in the resection group.

\section{Surgical complications}

The procedure-related complication rate was $4.5 \%$ in the resection group and $0 \%$ in the biopsy group. There was one $(1 / 22)$, postoperative intracerebral hematoma in the resection group which needed a re-operation.

\section{Neurological deterioration}

In the resection group we diagnosed neurological motor deficit or aphasia before surgery in 14 patients (64\%). Over one week after surgery, the neurological deficits disappeared in 4 patients, but new deficits developed in 3 patients (including one patient with postoperative hematoma). Three months after surgery, the neurological deficit had increased in 5 patients compared to before surgery $(23 \%)$. Including one patient with postoperative pneumonia died 1 month after surgery.

In the biopsy group we diagnosed neurological motor deficit or aphasia before surgery in 11 patients (69\%). Neurological deficits after biopsy developed slowly or had increased compare to before surgery over several weeks (1-3 months) in 15 cases (94\%). Including 9 patients died in this period of 3 months.

Worsening or new development of the permanent neurological deficits three months after surgery were significantly lower in the resection group (23\%), compared to the biopsy group (94\%), (Fisher's exact test, $P<0.0001$ ).

\section{KPS}

In the resection group, the median preoperative KPS was 80.0 (IQR 70-82.5) and three months after surgery it

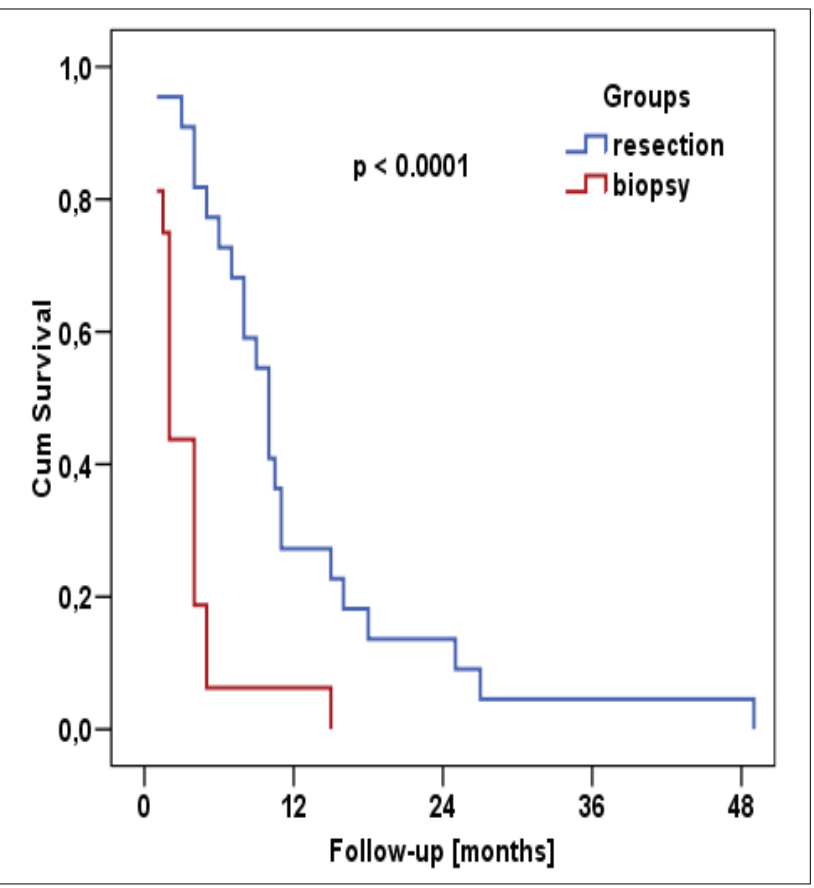

Fig. 1. The comparison of OS between the resection and the biopsy groups. was 80.0 (IQR 67.5-90.0). The difference was not statistically significant (Wilcoxon signed-rank test, $P=0.459$ ).

In the biopsy group, the median preoperative KPS and postoperative KPS (60.0, IQR 52.5-87.5) one week after procedure was unchanged.

\section{PS WHO}

In the resection group, three months after surgery, the median PS WHO was 1 (IQR 1-2). In the biopsy group, one week after surgery, the median PS WHO was 2 (IQR 1.25-2). The difference was not statistically significant (Mann-Whitney U-test, $P=0.154$ ).

\section{OS}

The mean overall survival time after resection was 12.2 months, 95\% CI was 7.8-16.6 months. The mean overall survival time after biopsy was 3.5 months, 95\% CI was 1.8-5.1 months (Fig. 1).

The difference in OS time between tumor resection group and biopsy group was highly statistically significant (log rank test, $P<0.0001)$.

\section{EOR}

In the resection group we evaluated GTR in 3 patients, NTR in 6 patients, STR in 7 patients and PR in 6 patients. The mean EOR was $90 \%$.

\section{EOR and OS}

In the resection group with EOR more than 95\% (9 patients) the median OS time was 11.0 months (95\% CI 9.6-12.4). In the resection group with EOR less than $95 \%$ (13 patients) the median OS time was 7.0 months (95\% CI 2.6-11.4). The difference in the mean overall survival time between these subgroups was not statistically significant.

\section{DISCUSSION}

GBM is the most frequent kind of brain tumor, accounting for approximately $12-15 \%$ of all intracranial neoplasms and $60-70 \%$ of astrocytic tumors. In most of Europe and North America, the incidence is in the range of 3-4 new cases per 100000 population per year. GBM may manifest at any age, but preferentially affects adults, with a peak incidence between 45 and 75 years of age ${ }^{2}$.

Standard treatment for GBM includes microsurgical resection followed by concomitant chemo-radiotherapy and then adjuvant chemotherapy. $\mathrm{O}^{6}$-Methylguanine-DNA methyltransferase (MGMT) is a repair protein that specifically removes promutagenic alkyl groups from the $\mathrm{O}^{6}$ position of guanine in DNA, thereby protecting cells against alkylating agents. MGMT promoter methylation is frequently present in GBM (45-75\%), and is associated with longer survival in GBM patients treated with temozolomide ${ }^{2}$.

Prognostic factors for patients with GBM include age, preoperative neurological function, EOR, and eloquent location $^{3}$. These factors, especially age and eloquent location, typically dictate the aggressiveness of the treatment strategy. Beyond establishing the histological diagnosis 
and decompressing tumor mass effect, the value of microsurgical resection of GBMs remains controversial ${ }^{4}$. A retrospective analysis of Lacroix et al. combining 416 patients with newly diagnosed and recurrent GBM concluded that more than $98 \%$ EOR is necessary to improve survival significantly ${ }^{1}$. This was the reason for indicating needle biopsy in cases of GTR preclusion, especially in EBA.

In recent years, with the advent of new surgical technologies (navigational systems, motor and language mapping, functional MRI including diffusion-tensor-imaging-based fiber tracking, intraoperative ultrasonography or MRI, intraoperative 5-ALA - mediated tumor fluorescence), there are new possibilities for achieving more extensive resection with the preservation of neurological function ${ }^{5,6}$. Awake craniotomy (AC) is mainly reserved for resection of tumors affecting the functionally eloquent cortex in order to reduce the risks of deficits as well ${ }^{7}$. New analysis of Sanai et al. supports the value of microsurgical resection for newly diagnosed GBMs when at least 78\% of the tumor volume can be resected. In cases in which this does not appear possible, tumor debulking remains the best option for alleviating symptoms due to mass effect and to establish a diagnosis. These data represent the largest reported volumetric outcome study for patients with newly diagnosed GBM, and suggest that EOR is a significant predictor of survival, even when a gross-total resection is not possible. For the 500 patients with GBM identified in Sanai et al., the median age was 60 years, and they presented with a median KPS score of 80 . The median preoperative tumor volume was $65.8 \mathrm{~cm}^{3}$ and most tumors (69\%) occupied an eloquent territory. All patients underwent image-guided microsurgical resection followed by chemotherapy and radiation therapy. The median postoperative tumor volume was $2.3 \mathrm{~cm}^{3}$, which equated to a $96 \%$ median EOR. The median OS was 12.2 months. A significant survival advantage was seen with as little as $78 \%$ EOR, which was associated with a 12.5 -month median survival, although the difference in median OS widened successively with higher EOR. An EOR more than $80 \%$ equated to a 12.8 -month median survival, whereas an EOR more than $90 \%$ led to a 13.8 -month median survival, and EOR of $100 \%$ carried a 16 -month median survival ${ }^{4}$. Similarly in our study OS time was associated with the EOR. In the subgroup with EOR more than 95\% the median OS was 11.0 months and in the subgroup with EOR less than $95 \%$ the median OS was only 7.0 months.

The most compelling evidence for EOR exists for low-grade gliomas and volumetric analyses have shown, that greater EOR suggest better OS, progression-free survival and malignant progression-free survival ${ }^{8}$. For highgrade gliomas the evidence is less consistent. Feigl et el. showed that GTR of GBM with 5-ALA in combination with monitoring could be achieved in $64 \%$ of the surgeries in eloquent areas ${ }^{9}$. Laws et al. compared the effect of surgery versus biopsy for patients (mean age 54 years) with malignant astrocytomas (GBM and anaplastic astrocytomas) and found that surgery was associated with longer survival than biopsy ${ }^{10}$. One prospective, randomized study does exist, comparing biopsy versus debulking for elderly patients (30 patients older than 65 years) with primary malignant glioma (grade III-IV). All the patients were referred to radiotherapy. The results indicated the survival benefit of resection ( 5.7 months) versus biopsy (2.8 months). Although, the study was unblinded and without adjuvant chemotherapy ${ }^{11}$. Chaichana et al. compared 133 and 72 consecutive patients aged 65 years and older who underwent surgery and needle biopsy for intracranial primary (de novo) GBM and were retrospectively reviewed. Patients who underwent resection had a median survival of 5.7 months compared with 4.0 months for patients who underwent needle biopsy. This study demonstrated that older patients tolerate aggressive surgery without increased surgery-related morbidity and have longer survival than similar patients undergoing needle biopsy ${ }^{3}$.

Not only OS but also time of deterioration and quality of life are important outcome measures if we discuss fatal disease. Vuorinen et al. suggested that there was 2.8 times higher risk for deterioration after biopsy than after craniotomy and resection ${ }^{11}$. Pereira et al. used AC and somato-sensory evoked potentials (SSEPs). They reported postsurgical recovery of previous motor deficits in $75 \%$ of patients, while motor deficits worsened in $9 \%$ of cases. In other reports the incidence of permanent deficits ranged from 0 to $16.9 \%$ after $\mathrm{AC}$ in EBA (ref. ${ }^{12}$ ).

Our report is the first prospective study, to our knowledge, that compares the results of resection and biopsy of primary GBM in EBA. The both groups were comparable, except less frequency of postoperative chemo-radiotherapy in the biopsy group compare to the resection group.

Accurate localization of eloquent cortex enables optimal neurosurgical tumor resection and minimizes post-operative neurological deficits. F-MRI is a non-invasive technique for examining brain function that utilizes changes in blood oxygenation to identify areas of increased or decreased neuronal activity. However, there are limitations because pre-operative localization is only as good as the patient's ability to perform the task or function one is attempting to localize ${ }^{13}$. Gonzáles et al. found that despite preoperative planning for multimodal navigation, motor f-MRI and subcortical pathways generated by diffusion tensor imaging (DTI) of intrinsic brain tumors located in motor eloquent areas and intraoperative neuromonitorization, the mean volumetric resection was $89 \%$. Preoperatively a total of $58.8 \%$ of patients had some motor deficit, increasing $24 \mathrm{~h}$ after surgery to $76.5 \%$ and decreasing to $41 \%$ a month later ${ }^{14}$. Patients with GBM and mild sensorimotor deficits had significantly less activation of the motor and sensory cortices on the side with the tumor than on the contralateral side on blood oxygen level-dependend (BOLD) f-MRI. This difference, which may be due to pressure effect or loss of vascular autoregulation, should be considered in preoperative planning in which BOLD f-MRI is used to identify eloquent cortices to be avoided during brain tumor surgery ${ }^{15}$. If the technology of preoperative f-MRI is applied indiscriminately to guide resection, especially in areas directly adjacent to areas of activation or within the motor gyrus itself, it may lead to resection of cortex that has lost the ability to be detected by BOLD f-MRI but that is still functioning ${ }^{12}$ ! 
This means that the limitation of these and all similar studies is identification of EBA. Brain anatomy, especially the gyrus topography, is characterized by a high interindividual variability, which implies insufficient prediction of functionality based on anatomical criteria. This is one reason for f-MRI for identification of motor and language EBA, but even this method is not completely reliable as mentioned above. More, the brain areas directly adjacent to the M1 and/or M2 segments of the middle cerebral artery have not traditionally been considered eloquent brain regions. From a surgical point of view, however, injury to these vessels can result in damage to the eloquent brain regions they supply ${ }^{16}$.

In our study, using quality of life and OS as criteria we found that there were clear advantages to resection of GBM over biopsy. For patients in good clinical condition and with tumors in or near EBA, recommended is resection of GBM with efforts to maximize EOR, despite the difference between the subgroups (less or more 95\% EOR) was not statistically significant in our study. Surgical resection in eloquent areas, besides prolonged survival and better quality of life, may also provide additional benefits beyond needle biopsy, including improved efficacy of radiotherapy and chemotherapy and less corticosteroid medication.

\section{CONCLUSION}

The mean extent of resection of GBM in eloquent brain areas was $90 \%$.

Worsening or new development of the permanent neurological deficits three months after surgery were significantly lower in the resection group (23\%), compared to the biopsy group (94\%).

The difference in overall survival time between tumor resection group (12.2 months) and biopsy group (3.5 months) was statistically significant.

For patients in good clinical condition and for tumors in or near eloquent brain areas resection of GBM, as radical as possible is recommended.

\section{ACKNOWLEDGEMENT}

Support in part by grant of IGA of Ministry of Health, Czech Republic No. NT11065-5/5/2010 and by grant of IGA of Ministry of Health, Czech Republic No. NT135814/2012(86-91).

Authorships contributions: All authors contributed equally to preparing the manuscript.

Conflict of interest statement: None declared.

\section{REFERENCES}

1. Lacroix M, Abi-Said D, Fourney DR, Gokaslan ZL, Shi W, DeMonte F, Lang FF, McCutcheon IE, Hassenbusch SJ, Holland E, Hess K, Michael C, Miller D, Sawaya R. A multivariate analysis of 416 patients with glioblastoma multiforme: prognosis, extent of resection, and survival. J Neurosurg 2001;95(2):190-8.

2. Louis DN, Ohgaki H, Wiestler OD, Cavenee WK (Eds.): WHO Classification of Tumours of the Central Nervous System. IARC: Lyon 2007.

3. Chaichana KL, Garzon-Muvdi T, Parker S, Weingart JD, Olivi A, Bennet $\mathrm{R}$, Brem H, Quinones-Hinojosa A. Supratentorial glioblastoma multiforme: the role of surgical resection versus biopsy among older patients. Ann Surg Oncol 2011;18(1):239-45.

4. Sanai N, Polley MY, McDermott MW, Parsa AT, Berger MS. An extent of resection threshold for newly diagnosed glioblastomas. J Neurosurg 2011;115:3-8.

5. Stummer W, Pichlmeier U, Meinel T, Wiestler OD, Zanella F, Reulen $\mathrm{HJ}$. Fluorescence-guided surgery with 5 -aminolevulinic acid for resection of malignant glioma: a randomized controlled multicentre phase III trial. Lancet Oncol 2006;7(5):392-401.

6. Stummer W, Reulen HJ, Pichlmeier U, Schumacher W, Tonn JC, Rohde V, Oppel F, Turowski B, Woiciechowsky C, Franz K, Pietsch T. Extent of resection and survival in glioblastoma multiforme: identification of and adjustment for bias. Neurosurgery 2008;62(3):654-76.

7. Sacko O, Lauwers-Cances V, Brauge D, Sesay M, Brenner A, Roux FE. Awake craniotomy vs surgery undr general anesthesia for resection of supratentorial lesions. Neurosurgery 2011;68(5):1192-8.

8. Sanai N, Berger MS. Glioma extent of resection and its impact on patient outcome. Neurosurgery 2008;62:753-66.

9. Feigl GC, Ritz R, Moraes M, Klein J, Ramina K, Gharabaghi A, Krischek B, Danz S, Bornemann A, Liebsch M, Tatagiba MS. Resection of malignant brain tumors in eloquent cortical areas: a new multimodal approach combining 5-aminolevulinic acid intraoperative monitoring. J Neurosurgery 2010;113(2):352-7.

10. Laws ER, Parney IF, Huang W, Anderson F, Morris AM, Asher A, Lillehei KO, Bernstein M, Brem H, Sloan A, Berger MS, Chang S. Survival following surgery and prognostic factors for recently diagnosed malignant glioma: data from the Glioma Outcomes Project. J Neurosurg 2003;99(3):467-73.

11. Vuorinen V, Hinkka S, Farkkila M, Jaaskelainen J. Debulking or biopsy of malignant glioma in elderly people - a randomized study. Acta Neurochir 2003;145:5-10.

12. Pereira LCM, Oliveira KM, Abbate GLL, Sugai R, Ferreira JA, Motta LA. Outcome of fully awake craniotomy for lesions near the eloquent cortex: analysis of a prospective surgical series of 79 supratentorial primary brain tumors with long follow-up. Acta Neurochir 2009;151:1215-30.

13. Zhang D, Johnston JM, Fox MD, Leuthardt EC, Grubb RL, Chicoine MR, Smyth MD, Snyder AZ, Raichle ME, Shimony JS. Preoperative sensorimotor mapping in brain tumor patients using spontaneous fluctuations in neuronal activity imaged with $\mathrm{fMRI}$ : initial experience. Neurosurgery 2009;65(6Suppl):226-36.

14. Gonzáles-Darder JM, Gonzáles-López P, Talamantes-Escribá F, Garciá-March G, Roldán-Badía P, Quilis-Quesada V, Verdú-López F, Bordes-García V, Botella-Maciá L, Masbout G, Cortés-Donate V, Belloch-Ugarte V. Treatment of instrinsic brain tumors located in motor eloquent areas. Results of a protocol based in navigation, tractography and neurophysiological monitoring of cortical and subcortical structures. Neurocirugia (Astur)2011;22(1):23-5.

15. Holodny Al, Schulder M, Liu WCh, Maldjian JA, Kalnin AJ. Decreased BOLD functional MR activation of the motor and sensory cortices adjacent to a glioblastoma multiforme: implications for image-guided neurosurgery. Am J Neuroradiol 1999;20:609-12.

16. Park JK, Hodges T, Arko L, Shen M, lacono DD, McNabb A, Bailey NO, KreisI TN, Iwamoto FM, Sul J, Auh S, Park GE, Fine HA, Black PM. Scale to predict survival after surgery for recurrent glioblastoma multiforme. J Clin Oncol 2010;28:3838-43. 